\title{
Pulmonary Rehabilitation - PR
}

\author{
Dh. Argjiri ${ }^{1}$, S. Bala ${ }^{1}$, F. Gradica ${ }^{1}$, A. Menzini ${ }^{1}$, L. Agolli ${ }^{1}$, V. Miha ${ }^{1}$, V. Selmani ${ }^{1}$, \\ O. Nuredini ${ }^{1}$, Y. Vakeflliu ${ }^{1}$ \\ University Hopital "Shefqet Ndroqi", Tirana, Albania \\ *Corresponding author:Dr.Dbimitraq Argiiri,dh.argizi@yahoo.com
}

\begin{abstract}
Chronic respiratory diseases are associated with severe, not only pulmonary, but also systemic damage such as dysfunction of peripheral muscles, dysfunction of respiratory muscles, nutritional disorders, cardiac injuries, skeletal disorders, sensory debilities, and psychological dysfunction. Mechanisms of these injuries are many and different. Pulmonary recovery (PR) is an integral part of clinical therapy in patients with chronic respiratory disease who continue to be symptomatic or continue to have pulmonary function depression, regardless of standard medical treatment.
\end{abstract}

Keywords: Chronic, respiratory, diseases, dysfunction, clinical therapy, pulmonary recovery 


\section{Introduction}

Chronic respiratory diseases are associated with severe, not only pulmonary, but also systemic damage such as dysfunction of peripheral muscles, dysfunction of respiratory muscles, nutritional disorders, cardiac injuries, skeletal disorders, sensory debilities, and psychological dysfunction. Mechanisms of these injuries are many and different. Pulmonary recovery (PR) is an integral part of clinical therapy in patients with chronic respiratory disease who continue to be symptomatic or continue to have pulmonary function depression, regardless of standard medical treatment.

Physical exercises and enlargement of the chest cavity were first described by Charles Deninson in 1895 in "Pulmonary Injuries Exercises". In the 20th century, Alvan Barach from New York, while studying the discovery, found that "the semi-stagnant attitude diminished the feeling of dispensation [1]. We do not yet have a proper pulmonary recovery services in our hospital.

\section{Discussion}

The Pulmonary Rehabilitation Committee of the American College of Chest Physicians in 1981 defined Pulmonary Rehabilitation as "An art of medical science where an individual multidisciplinary program, formulated on the basis of clear diagnosis, therapy, emotional support and education, stabilizes or restores psychological and psychopathological anomalies of pulmonary disease and strive to bring the patient to the highest possible functional level, allowing the pulmonary handicap and all life situations "[2,3].

Main goals of pulmonary rehabilitation:

1. Reduce the symptoms,

2. Reduce disability,

3. Increase participation in physical and social activities

4. Improve the quality of life (QOL) in patients with chronic respiratory disease.

These goals are achieved through patient and family education, exercise training, psychosocial intervention, lifestyle habits, and evaluation of outcomes. Pulmonary rehabilitation programs are individual for each patient and are implemented by a multidisciplinary medical practitioner.

\section{Patient Selection for Pulmonary Rehabilitation}

$P R$ is indicated for patients with chronic respiratory injuries, who, regardless of optimal medical treatment, continue to have dyspnea, decreased exercise tolerance, or restriction of life activity. The indication for PR is not based on pulmonary physiological damage, but on the persistence of symptoms, disability and handicap. The main respiratory pathologies that have the indications for pulmonary resuscitation programs are:

COPD, Asthma bronchialis, Thoracic Cage Diseases, Cystic fibrosis, Bronchiectasia, Defuse pulmonary diseases, Lung Cancer, Some neuromuscular diseases, Poliomyelitis syndrome, Postoperative conditions (thoracic and abdominal surgery, pulmonary transplantation, Lung volume reduction).

Currently, the COPD continues to be the main disease involved in PR programs. Exceptional criteria include situations that interfere with rehabilitation processes or have a risk of exercise training (cognitive dysfunction, severe pulmonary hypertension, unstable angina and myocardial infarction) [4].

\section{Application site for PR}

The implementation of pulmonary rehabilitation programs can be done:

In the hospital (inpatient or outpatient)

At home

The location choice depends on the physical, functional, psychological condition of the patient; the variability and distance from the recovery center and the patient's preference. Rehabilitation in the hospital is usually recommended for patients with severe injuries due to intensive rehabilitation services and available staff. Rehabilitation in outpatient conditions may be of interest to a large number of patients. Although preliminary results have not been well studied, home rehabilitation is more appropriate for patients and the family and can provide more motivation for continued training $[5,6]$. Regardless of where it is applied, the PR program, the effectiveness depends more on its structure and components 
rather than where it applies.

\section{Pulmonary Rehabilitation Staff:}

Because PR is a multidisciplinary complex of medical services, it is necessary to combine the experience of a multidisciplinary staff. The staff is run by the program director, who must be a physician pulmonologist with a medical-legal responsibility for treating the patient during rehabilitation. The Director is responsible for all aspects of the program:

Selection and involvement of patients; Requesting consensus and informing the patient in writing, Individualized selection; Controlling the quality of application to programs; Continue cure and programs from the general practitioner.

The program director should be trained in cardiopulmonary treatment; fitness exercises, ventilation, treatment of functional disorders, and to be able to work in the staff with other professionals.

Other Personnel: Nurse, Physiotherapist, who carry out the duties assigned by the doctor. A therapist for 5-6 patients in day-hospital and a therapist for very severe patients in Intensive Care Unit (mechanical ventilation) are needed.

Other staff members included:

\section{Coordinator}

2. Psychologist

3. Nutritionist

4. Social assistant

\section{Pulmonary recovery components}

PR programs generally have four major components:

Training

Education

Psychosocial intervention in the habits of life

Preliminary assessment

Muscle training is essential in PR programs. Exercise does not exacerbate the established pulmonary damage, but improves breathless and other parameters previously measured. Since the weakening of peripheral muscles contributes to the limitations of patients with pulmonary disease, muscle training is a component of training during PR. Lowering the muscular intensity of the upper and lower extremities may lead to decreased pulmonary ventilation.

Fig.1: Lower extremity exercises. A regular walking graph is an important component of PR. The described distance progressively increases and the supplaing of $\mathrm{O} 2$ is necessary in cases of hemoglobin desaturation.

Training of respiratory muscles, using appropriate methods improved their strength in patients with COPD. However, it remains unclear whether this improvement reduces symptoms, disability and handicap. [7, 8].

Education is an intégral part of RP programs. Education makes patients the most capable and cooperative in monitoring the disease and improves their compliance. Education is made with small groups or individuals and includes:

\section{Smoking cessation}

Education to save energy by breathing quietly;

Using the necessary auxiliary tools;

Education related to the medication used (Adaptation of Therapy);

Optimum use of inhalers (MDI, space - haler, etc.); Optimum use of 02 ;

Clarification of the risk of respiratory insufficiency;

Need for mechanical ventilation.

Smoking cessation continues to be the most important therapeutic intervention. Many patients with SPOK have a history of smoking or continue to use tobacco. Any tobacco smoking program should involve multiple interventions [9], to help the patient to quit smoking, to prevent the resumption of tobacco use and to cooperate with the family to understand the importance of smoking cessation. Nicotine replacement therapy can be used.

The respiratory control techniques and thoracic physiotherapy are two important components of pulmonary rehabilitation at COPD, bronchiectasia and cystic fibrosis. Although smoking cessation and long-term O2-therapy prolong the life of patients with COPD, physiotherapy appears to be the same in patients with bronchiektazy and cystic fibrosis [10]. The three main techniques used 
are :

\section{Half-closed lips expiration,}

Postural drainage techniques

Diaphragm breathing

Treatment with oral medications or inhalers is used in stable patients to reduce dyspnoea and increase exercise tolerance.

\section{Psychological intervention and lifestyle habits:}

Anxiety and depression are common in people with chronic pulmonary disease. Psychological intervention in the form of regular patient education, focusing on specifi

problems, is important. Instructing for muscle relaxation,

stress reduction and panic control, can help lessen sleepiness and anxiety. Due to the effect of chronic respiratory disease on family members, it is beneficial for family members or friends during the PR [11].

Depression is common in those with COPD. In this case the prevalence ranges from $20-60 \%$. Because of this are genetic predispositions, severe injuries and the effects of COPD on the SNQ.

Evaluation of the candidate for PR is necessary for the development of an individual treatment program.

Clinical history, physical examination, review of previous examinations (such as spirometry) are necessary to determine the degree of pulmonary damage. Determining basic exercise capacity is essential in determining exercises and evaluating hypoxemia during exercise. Evaluating the preliminary results is very important component of $\mathrm{PR}$ to determine individual patient response and evaluate the effectiveness of the program. The measurements to be performed before and after the patient rehabilitation program are:

\section{Dyspnoea}

Exercise skills

Medical condition

Activity level

Measurements should be carried out for a period of time of 6 to 12 months.
- Efforts and dyspnea:

- Discomfort is the most common symptom of patients with chronic pulmonary disease and major causes for medical help. Long-range dyspnea is usually measured with Borg scale or other analogous degrees. Effects during the daily activity are measured by the questionnaires described by the Medichal Research Council(mMRC). Decreasing the length of the exercise evidence indicates that PR has been effective.

Specific measurements for functional pulmonary condition:

- Functional capacity is what the patient is capable of doing, and functional performance is what the patient is currently doing every day.

- The functional reserve is the difference between them. Pulmonary Rehabilitation improves patient Functional capacity. [12,13]

Exercise Tests: The progressive effort test is carried out on a cyclo-ergometer or revolving tap. Exercise continues up to a maximum heart rate of $85 \%$. Long-lasting dispnea can be calculated using a visual analog scale. This test is reproducible, sensitive and can be improved by PR. A long lasting time shows longer training skills [14]. 6 minutes-walking test is well correlated with performance at peak speed. A clinically evaluated minimum increase is the $54 \mathrm{~m}$ long distance to this test.

Nutricional Evaluation: Weight loss in patients with chronic respiratory disease is a bad prognosis indicator. About $50 \%$ of COPD patients are suffering from protein and calcium malnutrition. The progressive fall in weight comes as a result of an inadequate diet, increased energy consumption, and inadequate breastfeeding. These mechanisms lead to energy disequilibrium and loss of body weight [15]. Improving nutrition during PR programs helps to improve the patient's health condition, the function of respiratory $\mathrm{mm}$. and above all the feeling of being good. Obesity, which is defined as $>30 \%$ of ideal BMI, may impair pulmonary function. The increase in fat mass increases the work of the respiratory system, which is now compromised. Intervention in a patient with malnutrition and chronic pulmonary 
disease consists in nutritional counseling, with the purpose of creating a planned and balanced diet and the use of supplemental foods.

Measurement of handicap and quality of life (QOL) [14]. For measuring the handicap and quality of life, these measuring instruments are used:

- General health questions

- Specific rates of disease, such as those of chronic respiratory disease.

\section{- St. George questionnaire}

- Specific disease-specific measurements

Some questionnaires can be used to determine anxiety and depression such as Diabetes Depression Inventory (DDI), geriarric depression scale (GDS).

\section{Benefits from Pulmonary Rehabilitation.}

The benefits of PR also appear in irreversible pulmonary diseases, as many of the disabilities result not from pulmonary damage but from the presence of secondary pathologies, which are often treatable if known. Although the rate of pulmonary obstruction in COPD does not change satisfactorily during pulmonary rehabilitation, it may be beneficial to improve muscle function and to walk for a longer distance with less dyspnea, increased independence, decreased hospital admissions, or dizziness with and improvement of QOL. Retrospective, studies have shown that there is no significant difference in improving tolerance to exercise or QOL after PR in patients with COPD versus non-COPD patients. So $P R$ is effective in patients with any disabilities, from any chronic respiratory disease and not just those with COPD [9].

Cost-effectiveness of $P R$

Although studies on the cost-effectiveness of PR describe it as relatively expensive, now they are considered not very accurate. A canadian study (2005), show that implementation of the COPD program for the community for a period of 1 year was accompanied by a reduction in hospital admissions, direct cost reduction and improvement of the health care system [16]. These are strong evidence demonstrating that pulmonary rehabilitation is a therapy that lowers the cost of treating chronic illnesses.

\section{In conclusion}

In our opinion, regarding the data gathered from various studies and our experience as well, we think that pulmonary rehabilitation is not only easy accesaable and doable program, but also a costoefective and with great inpact not only the pulmonary chronic diseases but it brings posistive effect in the whole body in general.

6. Lacasse Y, Wong E, Guyatt GH, et al. Metaanalysis of respiratory rehabilitation in chronic obstructive pulmonary disease. Lancet. Oct 26 1996;348(9035):1115-9

7. Bernard S, Whittom F, Leblanc P (1999) Aerobic and strength training in patients with chronic obstructive pulmonary disease. American Journal of Respiratory and Critical Care Medicine 159:896-901

8. Berry MJ, Adair NE, Sevensky KS, Quinby A, Lever HM (1996) Inspiratory muscle training and whole-body reconditioning in chronic obstructive pulmonary disease.

9. Puhan MA, Scharplatz M, Troosters T, Steurer J. Respiratory rehabilitation after acute exacerbation of COPD may reduce risk for readmission 
and mortality - a systematic review. Respir Res. 2005;6(1):54

10. Gosselink R. Breathing techniques in patients with chronic obstructive pulmonary disease (COPD). Chron Respir Dis. 2004;1(3):163-72

11. Norwood R. Prevalence and impact of depression in chronic obstructive pulmonary patients. Curr Opin Pulm Med. Mar 2006;12(2):113-7

12. Bestall JC, Paul EA, Garrod R, Garnham R, Jones PW, Wedzicha JA (1999) Usefulness of the Medical Research Council (MRC) dyspnoea scale as a measure of disability in patients with chronic obstructive pulmonary disease. Thorax 54:581586

13. California Pulmonary Rehabilitation Collabora- tive Group. Effects of pulmonary rehabilitation on dyspnea, quality of life, and healthcare costs in California. J

14. Smith K, Cook D, Guyatt GH, et al. Respiratory muscle training in chronic air flow limitation: a meta- analysis. ALYSIS. Mar 1992;145(3):533-9.

15. Gray-Donald K, Gibbons L, Shapiro SH, et al. Nutritional status and mortality in chronic obstructive pulmonary disease. Am J Respir Crit Care Med. Mar. 1996; 153(3):961-65

16. Maltais F, Bourbeau J, Lacasse Y, et al. A Canadian, multicentre, randomized clinical trial of homebased pulmonary rehabilitation in chronic obstructive pulmonary disease: rationale and methods. Can Respir J. May-Jun 2005;12(4):193-8. 